\title{
INVERSIÓN EN ENTRENAMIENTO EN EL EMPLEO: UNA REVISIÓN DE LA LITERATURA*
}

\author{
Martha Yánez Contreras ${ }^{* *}$ \\ Karina Acevedo González ${ }^{* * *}$
}

Recibido: mayo 19 de 2011 • Aceptado: junio 08 de 2012

\section{RESUMEN}

Este artículo hace una revisión sistemática de la literatura teórica y empírica que, desde la economía, analiza uno de los principales componentes del capital humano: el entrenamiento en el empleo. El análisis de la literatura sobre entrenamiento en el empleo permite evidenciar que los mayores desarrollos teóricos se han adelantado en el análisis de quién asume los costos del entrenamiento, mientras que los mayores desarrollos empíricos se han producido en el estudio de los efectos del entrenamiento sobre los salarios y la productividad, aunque la literatura del efecto en los salarios es mucho más robusta.

\section{PALABRAS CLAVE}

Entrenamiento en el empleo, imperfecciones en el mercado laboral, retorno de la inversión en entrenamiento, mercado laboral.

\section{CLASIFICACIÓN JEL} D92; J24; M53.

\section{CONTENIDO}

Introducción; 1. Entrenamiento general y específico; 2. Fuente de compresión de salarios: imperfecciones de mercado; 3. Retornos de la inversión en entrenamiento; 4. Conclusiones; Bibliografía.

\footnotetext{
* Este artículo de revisión de la literatura es resultado del proyecto de investigación "Retorno de la inversión en capacitación y entrenamiento de la pequeña empresa en Cartagena", adscrito al grupo de Mercado Laboral de la Universidad de Cartagena (categoría D de Colciencias) y financiado por dicha Universidad durante el período enero de 2011-enero de 2012.

** Economista, Universidad de Cartagena, Colombia. Magíster en Economía y magíster en Economía del Medio Ambiente y de los Recursos Naturales, Universidad de los Andes, Colombia. Profesora asociada Programa de Economía, Facultad de Ciencias Económicas, Universidad de Cartagena. Miembro del Grupo de Investigación en Mercado Laboral. Correo electrónico: myanezc@unicartagena.educ.co.

*** Economista, Universidad de Cartagena, Colombia. Joven investigadora del Grupo de Investigación de Mercado Laboral, Facultad de Ciencias Económicas, Universidad de Cartagena. Correo electrónico: kacevedog@unicartagena.edu.co.
} 


\title{
INVESTING IN EMPLOYMENT TRAINING: A LITERATURE REVIEW
}

\section{ABSTRACT}

This paper reviews the theoretical and empirical literature on Employment Training, which according to economic theory is one of the main components of human capital. The analysis of the literature on employment training provides evidence that the main theoretical developments that have been put forward have been around analyzing the party that pays for the training costs; while the main empirical developments have been around studying the effects of training on wages and productivity, even though the literature on the effect on wages is much more robust.

\section{KEY WORDS}

Employment training, labor market imperfections, return on investment for training, labor market.

\section{JEL CLASSIFICATION}

\author{
D92; J24; M53.
}

\section{CONTENT}

Introduction; 1 . General and specific training; 2. Source for salary compression: Market imperfections; 3. Return on investment in training; 4. Conclusions; Bibliography.

\section{INVESTIMENTO NO TREINAMENTO NO EMPREGO: UMA REVISÃO DE LITERATURA}

RESUMO

Este artigo faz uma revisão sistemática da literatura teórica e empírica, que desde a economia, analisa um dos principais componentes do capital humano: o treinamento no emprego. A análise da literatura sobre treinamento no emprego permite evidenciar que os maiores avanços teóricos tem se adiantado na análise de quem assume os custos do treinamento; enquanto que os maiores avanços empíricos tem se produzido no estudo dos efeitos do treinamento sobre os salários e a produtividade, porém a literatura do efeito nos salários é muito mais robusta.

\section{PALAVRAS CHAVES}

Treinamento no emprego, imperfeições no mercado laboral, retorno do investimento no treinamento, mercado laboral.

\section{CLASSIFICAÇÃO JEL}

D92; J24; M53.

\section{CONTEÚDO}

Introdução; 1. Treinamento geral e específico; 2. Fonte de compressão de salários: imperfeições de mercado; 3. Retornos do investimento no treinamento; 4. Conclusões; Bibliografia. 


\section{INTRODUCCIÓN}

Desde la publicación de los trabajos seminales de Becker (1962, 1964), Mincer (1957, 1962, 1974) y Schultz $(1961,1962)$ sobre el capital humano, la teoría económica ha destacado la importancia de la inversión en este factor en el aumento de la productividad y eficiencia laboral; de allí que ramas específicas de la economía hayan construido un sólido cuerpo teórico y empírico que estudia los retornos de esa inversión. No obstante, el análisis se ha concentrado en los retornos de la educación, marginándose el hecho de que en el proceso de adquisición de capital humano también intervienen los demandantes de trabajo o empleadores que de acuerdo con sus necesidades entrenan a los trabajadores en labores generales o específicas.

El estudio del entrenamiento data desde el mismo momento del surgimiento de la teoría moderna de capital humano. Desde entonces, el análisis ha girado en torno a quién asume los costos de entrenamiento y cuáles son los retornos de esa inversión. La primera inquietud, que se aborda desde un enfoque teórico, parte de la división hecha por Becker (1962) entre entrenamiento general y entrenamiento específico, mientras que la segunda, que se aborda esencialmente desde una perspectiva empírica, parte del análisis de Mincer (1962) sobre la contribución del entrenamiento a los salarios.

La distinción entre entrenamiento general y específico tiene interesantes implicaciones en la decisión de quién asume los costo de entrenamiento. El primer tipo de entrenamiento, según Becker (1962), incrementa la productividad tanto en la empresa que hace la inversión como en las restantes, mientras que el segundo, solo incrementa la productividad en la empresa que suministra el entrenamiento.

En condiciones de competencia perfecta, Becker (1962) plantea que las empresas no tienen incentivos para proporcionar entrenamiento general, ya que son los trabajadores y no las empresas quienes capturan el retorno de la inversión. No obstante, la literatura posterior demuestra que las empresas sí financian el entrenamiento general. Para ello se han derivado modelos alternativos que suponen la presencia de imperfecciones de mercado, entre ellas, información asimétrica, estructura institucional y costos de búsqueda y fricciones. Asimismo, se ha obtenido evidencia de que las empresas financian entrenamiento general al considerar mercados de competencia perfecta.

En cuanto al entrenamiento específico, el modelo de Becker (1962) plantea que la inversión es compartida entre los trabajadores y empleadores debido a que los retornos son compartidos. Si bien estas conclusiones fueron obtenidas bajo una estructura de mercado de competencia perfecta, las implicaciones se mantienen 
en mercados imperfectos, aunque en estos últimos es posible encontrar problemas de subinversión.

Por otro lado, el abordaje empírico del entrenamiento es una tarea compleja debido a que la medición del entrenamiento es aún un asunto en construcción y debido a la presencia de sesgo de selección en la muestra y endogeneidad. Según Barron, Black y Loewenstein (1997), la primera dificultad surge porque muchos trabajadores reciben entrenamiento de manera informal que no es cuantificable en forma directa; además, estos tienden a reportar menos intensidad de entrenamiento que los empleadores. Con respecto al sesgo de selección, se ha encontrado que la selección de trabajadores en entrenamiento está correlacionada con las habilidades inobservables de estos; de allí que el impacto en salarios y productividad no solo refleja el efecto del entrenamiento sino el efecto de las habilidades de los trabajadores.

En este orden de ideas, el objetivo del presente artículo es identificar el estado de la literatura sobre el entrenamiento en el empleo; por tanto, se presentan de una manera organizada los resultados de las investigaciones teóricas y empíricas sobre el tema, de modo que puedan ser usados para fines académicos y como una referencia para el campo empresarial en la toma de decisiones. Para esta revisión se hace una búsqueda sistemática en bases de datos electrónicas indexadas tales como Istor, ScienceDiret, EBSCOhost, Econlit, Scielo, Dialnet y Redalyc. En la búsqueda electrónica se usaron como descriptores los términos: on the job training, firm sponsored training, workplace training, en inglés y español, ya sea que se encuentren en todo el texto, en el resumen y/o en el título. La selección de los estudios se basó en la relevancia del título y/o resumen.

El presente artículo se divide en cuatro secciones, y se fundamenta en la discusión alrededor de la cual gira el estudio del entrenamiento: quién asume los costos y quién se apropia de los beneficios. En la primera sección, se aborda la división entre entrenamiento general y entrenamiento específico; en la segunda, se explica, a partir de las imperfecciones de mercado, el por qué las empresas financian el entrenamiento general; en la tercera sección, se presentan los estudios que analizan los retornos de la inversión en entrenamiento y, por último, se presentan las conclusiones.

\section{ENTRENAMIENTO GENERAL Y ESPECÍFICO}

El abordaje teórico del entrenamiento inicia con el trabajo seminal de Becker (1962) en el cual se distingue entre entrenamiento general y entrenamiento específico. El entrenamiento general, según este autor, tiene el potencial de incrementar la productividad tanto en la empresa que lo proveyó como en muchas otras, mientras 
que el entrenamiento específico solo es aprovechable por la empresa que realiza la inversión.

En un escenario de competencia perfecta, Becker (1962) plantea que las empresas no tienen incentivos para proveer entrenamiento general debido a que todo el retorno de la inversión sería apropiado por el trabajador en forma de un incremento en su salario, en igual proporción que el incremento en su productividad. En consecuencia, solo habrá entrenamiento general, siempre y cuando los trabajadores asuman todos los costos. En este orden de ideas, las empresas estarían dispuestas a proveer entrenamiento solo si los trabajadores aceptan salarios más bajos que su productividad durante el tiempo de entrenamiento'.

Conclusiones similares a las de Becker (1962) fueron esbozadas previamente por Pigou (1912), aunque este autor no distingue entre entrenamiento general y específico, y considera un mecanismo diferente por el cual las empresas no tienen incentivos para proveer entrenamiento: los trabajadores pueden renunciar para trabajar en otras empresas. Se destaca que para Becker (1962), la rotación laboral es independiente de la inversión de entrenamiento general, ya que los empleados asumen todos los costos y obtienen todos los retornos.

Con respecto al entrenamiento específico, el modelo de Becker (1962) plantea que la inversión es compartida entre los trabajadores y empleadores debido a que ambos comparten los retornos; sin embargo, estas inversiones compartidas ocurren cuando se espera que la relación laboral sea lo suficientemente larga para ambos. En particular, este autor postula que la decisión de cuánto invierte cada participante depende de la relación entre los salarios y la tasa de rotación laboral, beneficios y tasa de despido, y otros factores como la actitud frente al riesgo, los costos de financiamiento y las preferencias por la liquidez.

Por otra parte, Stevens (1994) amplía las conclusiones de Becker (1962) para un mercado imperfecto al derivar un modelo en el que las destrezas adquiridas en el entrenamiento son transferibles a un pequeño grupo de empresas (el caso intermedio de entrenamiento general y específico). Esta externalidad entre las empresas, que se incrementa con el tamaño del mercado (número de empresas competidoras), conduce a una inversión de entrenamiento por debajo del óptimo, ya que en este escenario la empresa que provee el entrenamiento captura cada vez menos los retornos de la inversión, y los empleados, cada vez más.

Contrario a los planteamientos de Becker (1962) que sugieren que las empresas no financian el entrenamiento general, en la literatura es posible encontrar un amplio

\footnotetext{
Al respecto, no existe una fuerte evidencia empírica que sugiera que el entrenamiento reduce los salarios iniciales. Los trabajos que aportan algunas evidencias empíricas sobre el tema son: Barron, Black y Loewenstein (1989); Barron, Berger y Black (1999); Bishop (1988) y Holzer (1990).
} 
grupo de trabajos que demuestran que las empresas financian al menos parte de esa inversión. Para evidenciarlo, se ha recurrido a las imperfecciones de mercado² que constituyen una fuente de distorsión de los salarios. El tipo de distorsión de salarios que ocurre cuando el efecto del entrenamiento en la empresa es mayor en la productividad que en los salarios es conocido en la literatura como salarios comprimidos.

Dentro de los autores que encuentran evidencia a favor de la hipótesis de los salarios comprimidos se destacan Brunello (2002), Bassanini y Brunello (2003), Booth y Zoega (2004), y Almeida-Santos y Mumford (2005). El primer autor encuentra evidencia de una relación positiva y significativa de los salarios comprimidos y el entrenamiento tanto general como específico para una muestra de 11 países europeos. Bassanini y Brunello (2003), con información de países de la Unión Europea y al medir la compresión de los salarios como el diferencial entre el crecimiento medio de los salarios de los empleados entrenados y no entrenados, encuentran una relación positiva entre la compresión de salarios y la incidencia del entrenamiento general. Almeida-Santos y Mumford (2005) demuestran que altos niveles de salarios comprimidos están relacionados de manera positiva tanto con la incidencia y duración del entrenamiento para Gran Bretaña. Por su parte, Booth y Zoega (2004) desarrollan un modelo que sugiere que la compresión relativa de los salarios incrementa el incentivo de invertir en entrenamiento general aunque no es una condición necesaria para que suceda.

Sin embargo, otros tratadistas encuentran que los salarios comprimidos no siempre inducen a las empresas a invertir. Peraita (2001), por ejemplo, muestra que a pesar de que España es uno de los mercados laborales más regulados registra una muy baja inversión en entrenamiento, lo que le permite concluir que una estructura de salarios muy comprimida necesariamente no proporciona mayores incentivos a las empresas para invertir en formación. Ericson (2008), por su parte, no encuentra una relación positiva entre los salarios comprimidos y el entrenamiento general, lo que el autor considera que no debe ser considerado como una negación de la teoría de los costos y beneficios compartidos del entrenamiento general. Esto indica que los trabajadores llevan un mayor costo del entrenamiento general que cuando es específico.

En síntesis, la división del entrenamiento en general y específico determina quién asume los costos de entrenamiento porque esta influye en quien se apropia de los beneficios. Si bien en la literatura de corte teórico, las empresas no financian entrenamiento general porque sus retornos son capturados por los trabajadores, la evidencia empírica muestra que las empresas sí realizan este tipo de inversiones,

Estas conclusiones también han sido encontradas considerando mercados perfectos como lo demuestra Balmaceda (2001) 
dada la existencia de imperfecciones de mercado que posibilitan que las empresas paguen salarios más bajos que sus productividades. Estas fuentes de imperfecciones de mercado son analizadas en la sección 2.

\section{FUENTE DE COMPRESIÓN DE SALARIOS: IMPERFECCIONES DE MERCADO}

De acuerdo con Acemoglu y Pischke (1999a y 1999b), en mercados imperfectos las empresas pagan a los trabajadores salarios más bajos que su productividad (salarios comprimidos). Estas diferencias surgen por diversas razones, entre estas, por costos de movilidad (fricciones de búsqueda y matching), asimetría de información y por la estructura institucional que caracteriza el sistema económico tal como la imposición de salarios mínimos y existencia de sindicatos.

\subsection{Asimetría de información}

La asimetría de información constituye la imperfección de mercado más estudiada en la literatura a la hora de explicar por qué las empresas proveen entrenamiento general. Según Acemoglu y Pischke, (1999a), hay dos tipos de informaciones que los potenciales empleadores pueden no tener: la cantidad de entrenamiento y el nivel de capital humano que el trabajador ha adquirido; y las habilidades de los trabajadores. Lo anterior crea un poder monopsónico del empleador actual en el sentido que conoce más las habilidades de los empleados y las características del entrenamiento proporcionado a estos que los empleadores potenciales.

La lógica detrás del porqué la asimetría de información incentiva a las empresas a invertir en entrenamiento general estriba en que esta distorsiona los salarios, ya que los potenciales empleadores, al no poder observar de manera directa la productividad del trabajador, ofrecerán salarios de acuerdo con la productividad esperada y no basada en la productividad efectiva, lo que, como consecuencia, interfiere en la posibilidad de que un trabajador se traslade de una empresa a otra. Para Waldman (1984), dado que las habilidades de los trabajadores son conocidas por el empleador actual, las empresas potenciales tratarán de reducir el problema al considerar el tipo de trabajo anterior como un proxy del nivel de habilidades del trabajador.

Con respecto a la asimetría de información que surge de la incertidumbre acerca de la cantidad de entrenamiento y nivel de capital humano que el empleado ha adquirido en los trabajos anteriores, Katz y Ziderman (1990) plantean que si los potenciales empleadores no tiene información sobre el alcance y tipo de entrenamiento general que se ha dado al trabajador, el beneficio que pueden obtener los trabajadores entrenados al moverse de una empresa a otra se reduce. Gibbons y Katz (1991) sugieren que las empresas podrían pagar por entrenamiento si esta 
inversión no es observable por las restantes. Chang y Wang (1996) muestran que la asimetría de información puede causar una distorsión en la inversión de capital humano, ya que la más alta productividad derivada del entrenamiento puede no ser reconocida por el mercado.

Por otra parte, la asimetría de información que se deriva de la no observancia de las habilidades de los empleados ha sido estudiada de manera amplia a partir del caso del sistema de aprendizaje. Al respecto, Acemoglu y Pischke (1998) elaboran su análisis al plantear que las empresas adiestradoras tienen más información de los aprendices que las empresas alternativas; por tanto, si un trabajador renuncia después de la graduación será tratado de igual forma que aquellos quienes han sido despedidos como de bajas capacidades. Esto reduce las oportunidades de los trabajadores retenidos y, en consecuencia, las empresas pueden obtener retornos del entrenamiento general al pagar un salario inferior a la productividad. Estos resultados fueron comprobados de manera empírica por los autores para el sistema de aprendizaje de Alemania.

Balmaceda y Sevilla (2001), al analizar el sistema de aprendizaje de Chile, sugieren que las empresas proveen entrenamiento general bajo asimetría de información y complementariedad estratégica entre entrenamiento general y específico al encontrar evidencia de que los aprendices retenidos tienen mayores salarios. Asimismo, Euwals y Winkelmann (2001), al usar información del sistema de aprendizaje de Alemania, encontraron que los aprendices que permanecen en la empresa de entrenamiento después de la graduación reciben más altos salarios y tienen una mayor duración en su primer empleo que aquellos que renuncian o son despedidos de la empresa.

Como se ha argumentado previamente, la asimetría de información interfiere en la posibilidad de que un trabajador se traslade de un trabajo a otro. Sin embargo, desde el punto de vista del empleador, en presencia de asimetría de información los beneficios derivados de la inversión en entrenamiento dependen de la tasa de retención de los empleados. Estos planteamientos difieren de los de la teoría estándar que considera ambas variables como independientes. Al respecto, Chang y Wang (1996) muestran que con asimetría de información las mayores tasas de rotación conducen a menor inversión en capital humano.

Los resultados de Chang y Wang (1996) fueron comprobados por Acemoglu y Pischke (1998) quienes plantean que la asimetría de información conduce a múltiples equilibrios: uno con alto entrenamiento y bajas tasas de rotación, dado que quienes renuncian tendrán bajos salarios; y otro, con bajo entrenamiento y alta rotación, ya que quienes renuncian reciben altos salarios y las empresas tienen moderado poder monopsónico. A diferencia de los autores anteriores, Stevens (1994b) desarrolla un modelo en el cual muestra que la asimetría de información incrementa la probabilidad 
de que un trabajador abandone la firma que entrena y aumenta la brecha entre los retornos privados y sociales.

Por otro lado, Hermalin (2002) desarrolla una extensión del modelo de Becker (1962) para explicar la subprovisión en entrenamiento general, lo que le permite sugerir que las empresas no proporcionarán entrenamiento con contratos a corto plazo pero la heterogeneidad en las habilidades de los trabajadores dificulta a la empresa el ofrecimiento de contratos con mayor horizonte temporal. No obstante, Acemoglu y Pischke (1999b) sugieren que los contratos a largo plazo o problemas en el mercado de crédito no son condiciones necesarias ni suficientes para que haya entrenamiento. Stevens (1994b), por su parte, sugiere que los contratos en los que la empresa escoge el salario resultan mejores en términos de reducir la asimetría de información y las externalidades, debido que le otorga menos ventaja a la empresa alternativa.

En contraposición a la extensa evidencia teórica y empírica que resalta la importancia de la asimetría de información en la explicación de la provisión de entrenamiento, Clark (2001) encuentra que no hay salarios diferenciales entre los retenidos y aquellos que renuncian después de graduados en el sistema de aprendizaje en Alemania; por tanto, rechaza la hipótesis de la asimetría de la información.

\subsection{Estructura institucional}

La teoría estándar de capital humano sugiere que la introducción del salario mínimo en un mercado competitivo reduce la inversión en entrenamiento general debido a que cubre a trabajadores que ya no pueden financiar tal entrenamiento a través de salarios más bajos. Dentro de los estudios empíricos que comprueban la hipótesis que el salario mínimo conduce a una menor inversión en entrenamiento se encuentran Leighton y Mincer (1981), Hashimoto (1982), Schiller (1994) y Neumark y Wascher (2001).

Contrario a los planteamientos de la teoría estándar y a los hallazgos de algunos trabajos empíricos, otro grupo de trabajos ha encontrado una débil fuerza del impacto negativo del salario mínimo sobre el entrenamiento. Al respecto, Grossberg y Sicilian (1999) no encontraron evidencia clara al usar información de cerca de 3.000 empresa de la encuesta Employment Opportunity Pilot Project (EOPP). Asimismo, Acemoglu y Pischke (2001), al usar información de National Longitudinal Survey of Youth (NLSY), no encuentran evidencia que el salario mínimo reduce el entrenamiento; por el contrario, encuentran evidencia que tiende a incrementarlo debido a que en mercados no competitivos el salario mínimo induce a las empresas a entrenar a los empleados no adiestrados. 
Resultados similares a los de Acemoglu y Pischke (2001) fueron encontrados por Arulampalam, Booth y Bryan (2004) quienes encuentran evidencia que la introducción del salario mínimo en Gran Bretaña aumentó la incidencia del entrenamiento. En particular, los autores encuentran que la probabilidad de entrenamiento se incrementó en un 8 y 10\% para los trabajadores afectados por la introducción del salario mínimo. También para Gran Bretaña, Metcalf (2004) plantea que la introducción del salario mínimo en estos países impulsó la probabilidad y la intensidad del entrenamiento. Asimismo, Fairris y Pedace (2004), al usar información de National Employer Survey (NES) y de la Standar Stadistical Establishment List (SSEL), no encuentran evidencia que el salario mínimo reduzca las horas promedio de entrenamiento y encuentran poca evidencia para sugerir que el salario mínimo reduce el porcentaje de trabajadores que reciben entrenamiento.

Además del salario mínimo, los sindicatos constituyen otra explicación que desde la estructura institucional se ha destacado para estudiar la provisión de entrenamiento general. Dustmann y Schonberg (2009), al usar información del programa de aprendizaje de Alemania, encuentran evidencia que los salarios son más comprimidos en empresas con sindicatos y que estas tienen más probabilidades de entrenar a los trabajadores en el programa de aprendizaje. Los resultados empíricos son consistentes con la hipótesis que los sindicatos mueven el entrenamiento más cerca al óptimo social, ya que ayudan a superar un tipo particular de falla de mercado: la inviabilidad de los contratos de salarios a largo plazo.

\subsection{Costos de movilidad: fricciones de búsqueda y matching}

Según Mortensen (1988), las limitaciones de movilidad (inercia residencial y fricciones de búsqueda y matching) al igual que la información asimétrica reducen las oportunidades de empleo de los trabajadores; en consecuencia, las empresas pueden pagar salarios por debajo de la productividad marginal del trabajador y así capturar rentas de la inversión en entrenamiento general. Al respecto, Shintoyo (2010) desarrolla un modelo de búsqueda y fricciones, el cual le permite sugerir que entre más fricciones prevalezcan en el mercado laboral mayor es la probabilidad de que las empresas financien entrenamiento general.

Una de las manifestaciones de la prevalencia de las fricciones de mercado es el desempleo: dado que la búsqueda de empleo requiere tiempo y recursos, se generan fricciones en el mercado, por eso puede haber desempleados a pesar de que existan vacantes. Sin embargo, De Paola y Scoppa (2002) encuentran que el entrenamiento está relacionado de manera negativa con el desempleo, ya que con alto desempleo las empresas tienden a contratar trabajadores adiestrados, más que proveer adiestramiento a los que no. Para extraer estas conclusiones estimaron modelos de búsqueda y fricciones matching para las regiones italianas. 
Esta hipótesis, al igual que la información asimétrica, ha sido estudiada a partir del programa de sistema de aprendizaje. Para Alemania, Harhoff y Kane (1997) encuentran que quienes abandonan el trabajo después del entrenamiento presentan mayores salarios. Estos resultados fueron respaldados por Clark (2001) también para Alemania. Este último autor estudia los costos de movilidad y la información asimétrica como fuente de entrenamiento.

\section{RETORNO DE LA INVERSIÓN EN ENTRENAMIENTO}

Mientras que los mayores desarrollos teóricos indagan por quién asume los costos del entrenamiento a partir de la división efectuada por Becker (1962), los mayores desarrollos empíricos se han adelantado en los efectos del entrenamiento sobre los salarios y la productividad que comenzó con el análisis de Mincer (1962). Esta literatura empírica deja de lado la discusión de quién realiza la inversión o quién se apropia de los retornos. Al respecto, Loewenstein y Spletzer (1997) no encontraron una diferencia sistemática entre los retornos de los salarios al entrenamiento general y específico.

\subsection{Sesgo de selección}

Un primer aspecto a considerar en el estudio empírico de los retornos de la inversión en entrenamiento es el sesgo de selección en la muestra que hace referencia a que la selección de trabajadores en entrenamiento está correlacionada con las habilidades inobservables de estos; de allí que el impacto en los salarios y en la productividad no solo refleja el efecto del entrenamiento sino también el efecto de las habilidades de los trabajadores. En particular, algunos estudios han encontrado que los más educados reciben más entrenamiento (Rosen, 1976; Veum, 1995; Blundell y otroso, 1999; Peraita, 2001)3.

Esta dificultad metodológica se ha superado al usar la corrección en dos etapas propuesta por Heckman (1979) y a través de la estimación de regresiones de efectos fijos; este último es el método de estimación más usado. No obstante, para Frazis y Loewenstein (2003) ambas metodologías tienen limitaciones: los efectos fijos, por ejemplo, no eliminan completamente el problema de heterogeneidad debido a que no controlan los efectos inobservables que son invariantes en el tiempo. Al respecto, Heckman, Ichimura y Todd (1997) encuentran que el sesgo de selección por características inobservables es menos importante que otros componentes. Por tanto, idean una extensión del método diferencias en diferencias, que resulta

\footnotetext{
3 No obstante, Ariga y Brunello (2006) encuentran que la educación y el entrenamiento muestran una relación negativa explicada porque el costo marginal es más alto para los más educados lo que ocurre debido a que el costo de oportunidad por el tiempo dedicado al entrenamiento se incrementa con la educación.
} 
efectivo para eliminar el sesgo de selección, en especial, cuando está afectado por componentes temporalmente invariantes, lo que supera la limitación del método de efectos fijos.

El trabajo de Frazis y Loewenstein (2003) muestra el potencial riesgo de no corregir por el sesgo de selección. Las estimaciones econométricas de estos autores arrojaron que la tasa de retorno en los salarios de 60 horas de entrenamientos son sustancialmente altas: se encuentran en un rango de 150\%-180\%. Sin embargo, al corregir por la heterogeneidad del crecimiento de los salarios (aquellos que con o sin entrenamiento muestran mayores tasas de crecimiento en los salarios) y por factores como los ascensos y los costos directos, estas tasas se reducen a cerca del $40 \%-50 \%$.

\subsection{Efecto en los salarios y productividad}

Si bien el abordaje empírico del entrenamiento es una tarea compleja debido al sesgo de selección, los resultados empíricos proporcionan una fuerte evidencia de la importancia de este factor en el incremento de la productividad y crecimiento de los salarios. Bartel (1995), por ejemplo, al tomar como unidad de análisis una gran empresa manufacturera estadounidense, encuentra que el entrenamiento tiene un efecto positivo y significativo sobre el crecimiento en salarios y productividad, incluso cuando se elimina el sesgo de selección. Resultados similares fueron encontrados por Alba-Ramírez (1994) para España, al usar una muestra de grandes y medianas empresas.

Se destaca que el estudio de los retornos de la inversión en entrenamiento ha estado concentrado principalmente en los retornos a los salarios; sin embargo, hay que precisar que si bien en condiciones de competencia perfecta los salarios reflejan completamente la productividad, esta situación no es similar con imperfecciones de mercado. De hecho, existe una fuerte evidencia empírica que sugiere que el efecto en la productividad es superior al efecto en los salarios (Bishop, 1996; Barron, Berger y Black, 1999; Acemoglu y Pischke, 1999a; Dearden, Reed y Van Reenen, 2005; Ballot, Fakhfakh y Taymaz, 2006).

Barron, Berger y Black (1999), al hacer uso de un modelo de efectos fijos para controlar las características inobservables y al emplear bases de datos de empleados (Small Bussiness Accounting -SBA) y empresas (Employment Opportunity Pilot Project -EOPP), encuentran que el crecimiento en los salarios está débilmente correlacionado con el entrenamiento, mientras que encuentran un impacto importante sobre el crecimiento de la productividad. Consistentes con estos resultados, también encuentran evidencia de que el impacto sobre la productividad es estadísticamente mayor al impacto en los salarios. 
Resultados similares fueron encontrados por Dearden, Reed y Van Reenen (2005) quienes, al analizar un panel de las industrias de Gran Bretaña entre 1983 y 1996 y al usar una variedad de técnicas de datos panel que incluye el método de momentos generalizados, encuentran que el efecto del entrenamiento en los salarios es cerca de la mitad del tamaño del efecto en la productividad; por tanto, las firmas obtienen los mayores rendimientos de la inversión. Estos autores también encuentran que un incremento en $5 \%$ de los trabajadores entrenados está asociado con un incremento del $4 \%$ en el valor agregado del trabajador y un aumento en 1,6\% en los salarios.

Conti (2005), por su parte, usa varias especificaciones y una variedad de datos panel para concluir que el entrenamiento impulsa de manera significativa la productividad; sin embargo, no encontró tal efecto en los salarios, por lo cual sugiere que las empresas recuperan la mayoría de los retornos. Ballot, Fakhfakh y Taymaz (2006) usan paneles de empresas de Francia y Suiza, y concluyen que los beneficios del entrenamiento son compartidos por las empresas y los trabajadores; sin embargo, son las empresas las que obtienen mayores retornos.

Estimaciones a largo plazo muestran un impacto pequeño del entrenamiento sobre los salarios. Al respecto, Kuruscu (2006) desarrolla una metodología no paramétrica para estimar las ganancias de ingresos a lo largo de la vida de la inversión en entrenamiento en el trabajo (OJT), lo que le permite sugerir que el incremento del ingreso a lo largo de la vida es menos del $1 \%$.

Por otra parte, Almeida y Carneiro (2008) estiman la tasa interna de retorno de la inversión de las grandes empresas (con más de 100 empleados) en Portugal con información detallada sobre la duración del entrenamiento, costo directo del entrenamiento y varias características de las empresas. Para controlar las características inobservables e invariables en el tiempo usan el método propuesto por Blundell y Bond (2000) que consiste en estimar las funciones de costo y de producción a partir de una aproximación de variables instrumentales en primeras diferencias implementado con un estimador del método generalizado de los momentos (GMM). Los resultados arrojan que para las empresas que proveen entrenamiento la tasa de retorno es de $8,6 \%$.

Bajo la misma línea de los autores anteriores, Lopes y Teixeira (2010) estiman la tasa interna de retorno de la inversión en entrenamiento de las grandes empresas en Portugal; sin embargo, a diferencia de Almeida y Carneiro (2008) calculan la tasa interna de retorno tanto para las empresas como para los trabajadores, y hacen la distinción entre costos directos e indirectos de entrenamiento. Los resultados muestran que la tasa interna de retorno para la empresa es de $11 \%$, mientras que la de los trabajadores es más alta, y se sitúa en 22\%. 
Como un segundo objetivo, Lopes y Teixeira (2010) desarrollan un modelo que examina los determinantes de la productividad y salarios de las empresas. Estos autores encuentran que una hora de entrenamiento por trabajador implica un incremento en 0,09\% en la productividad. Las ganancias de productividad son capturadas principalmente por las empresas, ya que estas se apropian de las $2 / 3$ de las ganancias, mientras que el 1/3 restante, por los trabajadores.

Se destaca que algunos estudios empíricos revelan que los rendimientos de la inversión en entrenamiento son decrecientes. Al respecto Greenhalgh y Stewart (1987) plantean que el beneficio marginal de una semana adicional cae a cero una vez el individuo ha acumulado cuatro semanas de entrenamiento. También encuentra evidencia que las destrezas adquiridas se deprecian dentro de una década o más. Estos resultados son respaldados por Frazis y Loewenstein (2003) quien encuentra que los retornos de una hora adicional de entrenamiento disminuyen con la cantidad de entrenamiento recibida.

Existen ciertas características que interfieren en la magnitud del impacto del retorno de la inversión como la educación, el género, el estado civil y la edad. Grossberg y Sicilian (1999) encuentran que la tasa de retorno de la inversión en entrenamiento es alta y se incrementa con la educación: la tasa de retorno de los menos educados es de 13\%, quienes tienen una educación intermedia tienen una tasa de 36\%, mientras que los de mayor educación tienen una tasa del $80 \%$.

Parent (2003), al usar un modelo de efectos fijos, encuentra que el entrenamiento tiene un impacto considerable sobre los salarios de los hombres, mientras que el efecto es mucho más modesto para las mujeres, resultados opuestos a los de Budria y Telhado (2004) quienes encuentran que las mujeres obtienen mayores retornos. Estos autores, al usar la encuesta Portuguese Labour Force Survey, estiman que los retornos en los salarios son cerca del $12 \%$ para los hombres, y $37 \%$, en el caso de las mujeres. Además, encuentran que los individuos menos educados y con alta experiencia profesional obtienen mayores salarios. En un estudio posterior, Budria y Telhado (2007) encuentran resultados similares, aunque esta vez los retornos para las mujeres fueron de $38 \%$ y de $30 \%$ para los hombres.

La literatura también ha examinado el impacto diferenciado de los distintos tipos de entrenamiento. Al respecto, Veum (1995) investiga el impacto en los salarios de los programas de entrenamiento de las empresas, del sistema de aprendizaje, de las escuelas de negocios y de las instituciones técnicas y vocacionales. Este autor sugiere que la incidencia de los programas de entrenamiento de las empresas y los seminarios fuera del lugar de trabajo está relacionada de forma positiva con el nivel de salarios, así como con los incrementos en estos entre 1986 y 1990. Para hacer 
frente al sesgo de selección aplica el procedimiento de corrección en dos etapas, y las primeras diferencias del logaritmo de los salarios.

Barrett y OConnell (2001) buscan estimar los efectos del entrenamiento específico y general sobre el crecimiento de la productividad en las empresas. Encuentran un impacto positivo y significativo del entrenamiento sobre el crecimiento de la productividad (solo el general). Este resultado se mantiene si se controla por factores como tamaño de la empresa, nivel inicial de capital humano, cambios en la organización y reestructuración.

Por otro lado, Frazis y Loewenstein (2003) examinan diferentes formas funcionales para analizar los retornos en los salarios, lo que les permite sugerir que las especificaciones en raíz cúbica y en logaritmos ajustan mejor los datos.

Por último, contrario a lo encontrado por numerosos análisis empíricos que demuestran el impacto positivo del entrenamiento sobre la productividad, Black y Lynch (2001) no encontraron relación entre estas dos variables.

\section{CONCLUSIONES}

El estudio de la teoría moderna del capital humano, que toma fuerza a partir de la década de los sesenta, ha conformado un robusto cuerpo teórico y empírico que evidencia la importancia de este factor en la productividad laboral. No obstante, el análisis del capital humano se ha centrado en la educación, a pesar de que la inversión en entrenamiento en las empresas tiene características mucho más deseables.

El estudio del entrenamiento ha girado en torno a dos grandes líneas de investigación, a saber: quién asume los costos de entrenamiento, que se aborda principalmente desde un enfoque teórico; y cuáles son los retornos de esta inversión, abordado desde una perspectiva empírica.

La primera línea de interés parte de la distinción realizada por Becker (1962) entre entrenamiento general y entrenamiento específico, desde el cual se deriva un amplio grupo de trabajos que intentan probar la hipótesis de que las empresas sí invierten en entrenamiento general. Para esto, los tratadistas han recurrido a estudiar el efecto de la compresión de los salarios que resulta de la existencia de imperfecciones de mercado. Entre estas imperfecciones de mercado se encuentran la información asimétrica, la estructura institucional (presencia de salarios mínimos y sindicatos) y los costos de movilidad.

Por otro lado, el estudio empírico se centra en el impacto del entrenamiento en la productividad y los salarios. Al respecto, existe una fuerte evidencia de que el efecto sobre estas dos variables es positivo, aunque algunos tratadistas han encontrado que el impacto sobre la productividad es superior al de los salarios. Las estimaciones 
econométricas en este sentido usan regresiones de efectos fijos para controlar las características inobservables y así hacer frente al sesgo de selección. No obstante, han surgido métodos cada vez más completos para hacer frente a este problema.

\section{BIBLIOGRAFÍA}

Acemoglu, Daron y Pischke, Jorn-Steffen (1998). Why Do Firms Train? Theory and Evidence. En: The Quarterly Journal of Economics, Vol. 113, No. 1, p. 79-119.

Acemoglu, Daron y Pischke, Jorn-Steffen (1999a). Beyond Becker: Training in Imperfect Labour Markets. En: Economic Journal, Vol. 109, No. 453, p. 112-142.

Acemoglu, Daron y Pischke, Jorn-Steffen (1999b) The structure of wages and investment in general training. En:Journal of Political Economy, Vol. 107, No. 3, p. 539-572.

Acemoglu, Daron y Pischke, Jorn-Steffen (2001). Minimum Wages and On-the-Job Training. En: IZA Discussion Paper, No. 384, 47p.

Alba-Ramirez, Alfonso (1994). Formal Training, Temporary Contracts, Productivity and Wages in Spain. En: Oxford Bulletin of Economics and Statistics, Vol. 56, No. 2, p. 151-170.

Almeida, Rita y Carneiro, Pedro (2008). The return to firm investments in human capital. En: Social Protection Discussion Papers 44947, The World Bank, 32p.

Almeida-Santos, Filipe y Mumford, Karen (2005). Employee training and wage compression in Britain. En: Manchester School, University of Manchester, Vol. 73, No. 3, p. 321-342.

Ariga, Kenn y Brunello, Giorgio (2006). Are Education and Training Always Complements? Evidence from Thailand. En: Industrial and Labor Relations Review, Vol. 59, No. 4, p. 613-29.

Arulampalam, Wiji; Booth, Alison y Bryan, Mark (2004).Training and the New Minimum Wage. En: The Economic Journal, Vol. 114, No. 494, Conference Papers, p. C87-C94.

Ballot, Gérard; Fakhfakh, Fathi y Taymaz, Erol (2006). Who Benefits from Training and RED, the Firm or the Workers? En: British Journal of Industrial Relations, London School of Economics, Vol. 44, No. 3, p. 473-495.

Balmaceda, Felipe (2001). Firm-Sponsored General Training in a Frictionless Labor Market. En: Ilades-Georgetown, Working Paper I-139, 35p.

Balmaceda, Felipe y Sevilla María (2001). Invirtiendo en entrenamiento general: el programa de Formación dual. En: Revista de análisis económico, Vol. 16, No. 2, p. 137-154.

Barrett, Alan y OConnell Philip (2001). Does Training Generally Work? The Returns to In-Company Training. En: Industrial and Labor Relations Review, Vol. 54, No. 3.

Barron, John; Black, Dan y Loewenstein, Mark (1989). Job Matching and On-the-Job Training. En: Journal of Labor Economic, Vol. 7, No. 1, p. 1-19.

Barron, John; Black, Dan y Loewenstein, Mark (1997). How Well Do We Measure Training? En: Journal of Labor Economics, Vol. 15, No 3, p. 507-528

Barron, John M.; Berger, Mark C.; Black, Dan A. (1999). Do Workers Pay for On-the-Job Training? En: Journal of Human Resources, Vol. 34, No. 2, p. 235-52. 
Bartel, Ann (1995). Training, Wage Growth, and Job Performance: Evidence from a Company Database. En: Journal of Labor Economics, Vol. 13, No. 3, p. 401-25.

Bassanini, Andrea y Brunello, Giorgio (2003). Is training more frequent when wage compression is higher? Evidence from European Community Household Panel. En: IZA Discussion Paper, No. 839,41 p.

Becker, Gary (1962). Investment in Human Capital: A Theoretical Analysis. En: Journal of Political Economy, Supplement, Vol. 70, p. 9-49.

Becker, Gary (1964). Human Capital. New York. Columbia University Press for the National Bureau of Economic Research, 412p.

Bishop, John (1988). Do Employers Share the Costs and Benefits of General Training? En: Working Paper \#88-08, Cornell University Center for Advanced Human Resource Studies, 32p.

Bishop, John (1996). What We Know About Employer-Provided Training: A Review of the Literature. En: Working Paper \#96-09, Cornell University Center for Advanced Human Resource Studies. New York: Cornell University, 57p.

Black, Sandra y Lynch, Lisa (2001). How To Compete: The Impact Of Workplace Practices And Information Technology On Productivity. En: Review of Economics and Statistics, Vol. 83, No. 3, p. 434-445.

Blundell, Richard; Dearden, Lorraine; Meghir, Costas y Sianesi, Barbara (1999). Human Capital Investment: The Returns from Education and Training to the Individual, the Firm and the Economy. En: Fiscal Studies, Vol. 20, No. 1, p. 1-23.

Blundell, Richard y Bond, Steve (2000). GMM Estimation with Persistent Panel Data: An Application to Production Functions. En: Econometric Reviews, Vol. 19, 24p.

Booth, Alison y Zoega, Gylfi (2004). Is Wage Compression a Necessary Condition for Firm-Financed General Training? En: Oxford Economic Papers, New Series, Vol. 56, No. 1, p. 88-97.

Budría, Santiago y Telhado, Pedro (2004). On the Returns to Training in Portugal. En: Discussion Paper 1429, Institute for the Study of Labour (IZA), 45p.

Budría, Santiago y Telhado, Pedro (2007). The wage effects of training in Portugal: differences across skill groups, genders, sectors and training types. En: Applied Economics, Taylor and Francis Journals, Vol. 39, No. 6, p. 787-807.

Brunello, Giorgio (2002). Is training more frequent when wage compression is higher? Evidence from 11 European countries. En: FEEM Working Paper No. 10, 13p.

Chang, Chun y Wang, Yijiang (1996). Human Capital Investment under Asymmetric Information: The Pigovian Conjecture Revisited. En: Journal of Labor Economics, Vol. 14, No. 3, p. 505-519.

Clark, Damon (2001). Why do German firms subsidize apprenticeship training? Tests of the asymmetric information and mobility cost explanations. En: Vierteljahreshefte zur Wirtschaftsforschung, 1/2001, p. 102-106.

Conti, Gabriella (2005). Training, productivity and wages in Italy. En: Labour Economics, Vol. 12, p. 557-576. 
De Paola, Maria y Scoppa, Vincenzo (2002). Firms' training decisions and unemployment in italian labour markets. En: Giornale degli Economisti e Annali di Economia, Vol. 61, No. 1, p. 103-126.

Dearden, Lorraine; Reed, Howard y Van Reenen, John (2005). Who gains when workers train? Training and corporate productivity in a Panel of British Industries. En: Oxford Bulletin of Economics and Statistics, forthcoming, 69p.

Dustmann, Christian y Schonberg, Uta (2009). Training and Union Wages. En: Review of Economics and Statistics, Vol. 91, No. 2, p. 363-76.

Ericson, Thomas (2008). The Effects of Wage Compression on General and Firm-Specific Training. En: Applied Economics Letters, Vol. 15, No. 1-3, p. 165-69

Euwals, Rob y Winkelmann, Rainer (2001). Why do Firms Train? Empirical Evidence on the First Labour Market Outcomes of Graduated Apprentices. En: IZA Discussion Paper 319, 41p.

Fairris, David y Pedace, Roberto (2004). The Impact of Minimum Wages on Job Training: An Empirical Exploration with Establishment Data. En: Southern Economic Journal, Vol. 70, No. 3, p. 566-83.

Frazis, Harley y Loewenstein, Mark (2003). Reexamining the Returns to Training: Functional Form, Magnitude, and Interpretation. En: BLS Working Paper 367, 69p.

Gibbons, Robert y Katz Lawrence (1991). Layoffs and Lemons. En: Journal of Labor Economics IX, p. 351-380.

Greenhalgh, Christine y Stewart, Mark (1987), The effects and determinants of training. En: Oxford Bulletin of Economics and Statistics, Vol. 49, p. 171-190.

Grossberg, Adam y Sicilian, Paul (1999). Minimum Wages, On-the-Job Training, and Wage Growth. En: Southern Economic Journal, Vol. 65, No. 3, p. 539-56.

Harhoff, Dietmar y Kane, Thomas (1997). Is the German Apprenticeship System a Panacea for the US Labor Market? En: Journal of Population Economics, Vol. 10, p. 171-196.

Hashimoto, Masanori (1982). Minimum Wage Effects on Training on the Job. En: American Economic Review, American Economic Association, Vol. 72, No. 5, p. 1070-87.

Heckman, James (1979). Sample Selection Bias as a Specification Error. En: Econometrica, Vol. 47, No. 1, p. 153-161.

Heckman, James; Ichimura, Hidehiko y Todd, Petra (1997). Matching as an Econometric Evaluation Estimator: Evidence from Evaluating a Job Training Programme. En: Review of Economic Studies, Vol. 64, No. 4, p. 605-54.

Hermalin, Benjamin (2002). Adverse Selection, Short-Term Contracting, and the Underprovision of On-the-Job Training. En: Contributions to Economic Analysis and Policy, Vol. 1, No. 1, 22p.

Holzer, Harry (1990). The Determinants of Employee Productivity and Earnings: Some New Evidence. En: Industrial Relations, Vol. 29, No. 3, p. 403-22.

Katz, Eliakim y Ziderman, Adrian (1990). Investment in General Training: The Role of Information and Labour Mobility. En: The Economic Journal, Vol. 100, No. 403, p. 1147-1158. 
Kuruscu, Burhanettin (2006). Training and Lifetime Income. En: The American Economic Review, Vol. 96, No. 3, p. 832-846.

Leighton, Linda y Mincer, Jacob (1981). The Effect of Minimum Wages on Human Capital Formation. En Rottenberg, Simon (editor): The Economics of Legal Minimum Wages, Washington, American Enterprise Institute for Public Policy Research, 155-173.

Loewenstein, Mark y Spletzer, James (1997). General and Specific Training: Evidence and Implications. En: Journal of Human Resources, Vol. 34, No. 4, p. 710-733.

Lopes, Ana Sofia y Teixeira, Paulino (2010). Productivity, wages and the returns to firm-providing training: who is grabbing the biggest share? Instituto Politécnico de Leiria y Universidade de Coimbra, Portugal, 29p.

Metcalf, David (2004). The impact of the national minimum wage on the pay distribution, employment and training. En: Economic Journal, Vol. 114, No. 494, p. C84-C86.

Mincer, Jacob (1957). A Study on Personal Income Distribution. New York, Columbia University, 306p.

Mincer, Jacob (1962). On-the-job Training: Costs, Returns, and Some Implications. En: The Journal of Political Economy, Vol. 70, p. 550-579.

Mincer, Jacob (1974). Schooling experience and earnings. New York, Columbia University Press for the National Bureau of Economic Research, 95p.

Mortensen, Dale (1988). Wages, Separations, and Job Tenure: On-the-Job Specific Training or Matching? En: Journal of Labor Economics, Vol. 6, No. 4, p. 445-

Neumark, David y Wascher, William (2001). Minimum Wages and Training Revisited. En: Journal of Labor Economics, Vol. 19, No. 3, p. 563-95.

Parent, Daniel (2003). Employer-Supported Training in Canada and Its Impact on Mobility and Wages. En: Empirical Economics, Vol. 28, No. 3, p. 431-59.

Peraita, Carlos (2001). Firm Sponsored Training In Regulated Labor Markets: Evidence From Spain. En: Working Papers. Serie EC 2001-15, Instituto Valenciano de Investigaciones Económicas,

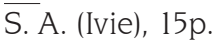

Pigou, Arthur. (1912). Wealth and welfare. London, Macmillan, 544p.

Rosen, Sherwin (1976). A Theory of Life Earnings. Journal of Political Economy, En: Vol. 84, No. 4, p. S45-67.

Schiller, Bradley (1994). Moving up: the training and wage gains of minimum wage entrants. En: Social Science Quarterly, Vol. 75, No. 3, p. 622-36.

Schultz, Theodore (1961). Investment in Human Capital. En: The American Economic Review, Vol. 2, p. 1-17.

Schultz, Theodore (1962). Investment in Human Beings. En: Journal of Political Economy, Vol. 70, p. 51-515.

Shintoyo, Naoki (2010). Worker-financed versus Firm-sponsored Training: How are Skilled Workers Supplied to the Economy? En: Economica Vol. 77, No, p. 110-127. 
Stevens, Margaret (1994). A Theoretical Model of On-the-Job Training with Imperfect Competition. En: Oxford Economic Papers, New Series, Vol. 46, No. 4, p. 537-562.

Stevens, Margaret (1994b). Labour Contracts and Efficiency in on-the-Job Training. En: The Economic Journal, Vol. 104, No. 423, p. 408-419.

Veum, Jonathan (1995). Sources of Training and Their Impact on Wages. En: Industrial and Labor Relations Review, Vol. 48, No. 4, p. 812-26.

Waldman, Michael (1984). Job Assignments, Signalling and Efficiency. En: The RAND Journal of Economics, Vol. 15, No. 2, p. 255-267. 